\title{
Acoustic Emission Wavelet Analysis of Filament Wound Composite Tube Fatigue Damage Process
}

\author{
Su Hua* and Chang Cheng
}

Tianjin Polytechnic University, Binshui Road 399, Xiqing District, Tianjin, 300387, P.R. China

\begin{abstract}
This paper performed a radial compression fatigue test on glass fiber winding composite tubes, collected acoustic emission signals at different fatigue damages stages, used time frequency analysis techniques for modern wavelet transform, and analyzed the wave form and frequency characteristics of fatigue damaged acoustic emission signals. Three main frequency bands of acoustic emission signal had been identified: $80-160 \mathrm{kHz}$ (low frequency band), 160-300 kHz (middle frequency band), and over $300 \mathrm{kHz}$ (high frequency band), corresponding to the three basic damage modes: the fragmentation of matrix resin, the layered damage of fiber and matrix, and the fracture of cellosilk respectively. The usage of wavelet transform enabled the separation of fatigue damaged acoustic emission signals from interference wave, and the access to characteristics of high signal-noise-ratio fatigue damage.
\end{abstract}

Keywords: Acoustic emission test, fatigue damage, filament winding composite tube, radial compression, wavelet transform.

\section{INTRODUCTION}

As a new dynamic detection technology, the acoustic emission measurement technology has the unique advantage of being "dynamic", 'real-time', "whole", "on-line", and so on. It can not only determine the seriousness of injury and the position of fault, but also predict the performance life of the specimen. Acoustic emission is also known as stress wave emission, referring to the phenomena that parts of the materials emit instantaneous elastic waves due to the rapid release of energy [1]. The technology of using acoustic emission detection system to gather, record and analyze acoustic emission signals and evaluate the nature of the acoustic emission source is called acoustic emission technology [2]. The unique advantages of acoustic emission technique are that it is able to make real-time, on-line, and non-destructive testing, and it also has high sensitivity [3, 4]. Therefore, the acoustic emission technique is widely used in detecting fatigue damage for a variety of materials. Filament winding composite tube, with the merits of light weight, high strength, corrosion resistance, good insulating properties and thermal performance, is widely used in space engineering that requires for high performance and low weight. This composite material consists of various materials and will damage and deform greatly under load. As a result, accurate deformation analysis for this kind of structure becomes very important.

Many scholars have done a great amount of research on the fatigue damage mechanism of filament winding structure under internal and external pressure. Mehan, R. L. et al. have done earlier study and suggested to use spectrum analysis to investigate the damage characteristics of the material [5], but

\footnotetext{
*Address correspondence to this author at the School of Computer Science \& Software Engineering, Tianjin Polytechnic University, Binshui Road 399, Xiqing District, Tianjin, 300387, P.R. China; Tel: +86 13821222528; E-mail: hua_207@126.com
}

the main difficulty is that signals with different patterns were often simultaneous but also indistinguishable. Giordano M. et al. made use of the observation of polarized light microscope and trigger effects of resonant transducer, applying broadband sensors to record the acoustic emission signals of breaking single fiber when carbon fiber thermoset composites were stretched, using FFT to describe its spectrum characteristics, comparing the signals from different stretching tests and then analyzing them to point out that the breakage of fiber has obvious frequency spectrum characteristics. Johnson M. et al. used broadband sensors to study the different acoustic emission signals of glass fiber/epoxy composite laminates when stretched on different plies, pointing out that different damage modes have different injury amplitudes, duration, and frequency range, and also categorizing the signals into several main types to differentiate different kinds of damage [6]. Amilcar Q. et al. studied the acoustic emission signals of the "sandwich" carbon fiber reinforced composite during the process of static stretching and fatigue test, pointing out that loading conditions have significant effects on signal characteristics [7]. Daenis, Y. et al. used the method of AE signals classification and multi-parameter filtering to record and analyze the damage evolution process of graphite/epoxy composite laminates when stretched [8]. H.Y.Chou. et al. continuously and circularly exerted pressure on carbon fiber composite vessel, using acoustic emission technique to detect and research on this type of vessel damage [9]. In addition, there are a large number of studies using $\mathrm{AE}$ to analyze the damage process of carbon fiber and glass fiber composite [10-12]. However, under the influence of factors such as material properties and test environment, $\mathrm{AE}$ wave is often shown as a complex, non-stationary, and random signal, containing a variety of noise. Therefore, finding an effective method of signal analysis is particularly important. Wavelet analysis uses a family of wavelet based functions to 
represent or approach signals and solves the conflicts of time-frequency resolution.

Through exerting radial pressure on multi-directional filament winding tube, wavelet transforming the AE signals collected in the course of damage, and filtering out noise signals among the original signals, this paper improves the accuracy of damage mechanism and sound localization when analyzing and differentiating fiber breakage, matrix cracking, and delamination of materials, and also provides study foundation for the early detection of mild damage

\section{TEST SPECIMEN AND METHOD}

\subsection{Multi-Directional Filament Winding Material}

Multi-directional filament winding composite is a kind of product that is formed by spiral winding, circular winding and axial winding, whose basic line schema is shown in Fig. (1). This product features in achieving pure axial winding, i.e. the winding angle $\alpha=0^{\circ}$. Compared with the traditional products formed by only spiral winding and circular winding, this structure can better withstand axial load and is easier to achieve iso-strength and iso-stiffness design, so it is widely applied.

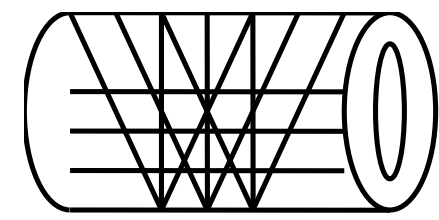

Fig. (1). Basic pattern of a multi-directional filament winding material tube.

Using E2 glass fiber and epoxy resin as raw materials, laminated structure and geometrical parameters are shown in Table 1. Test product is shown in Fig. (2).

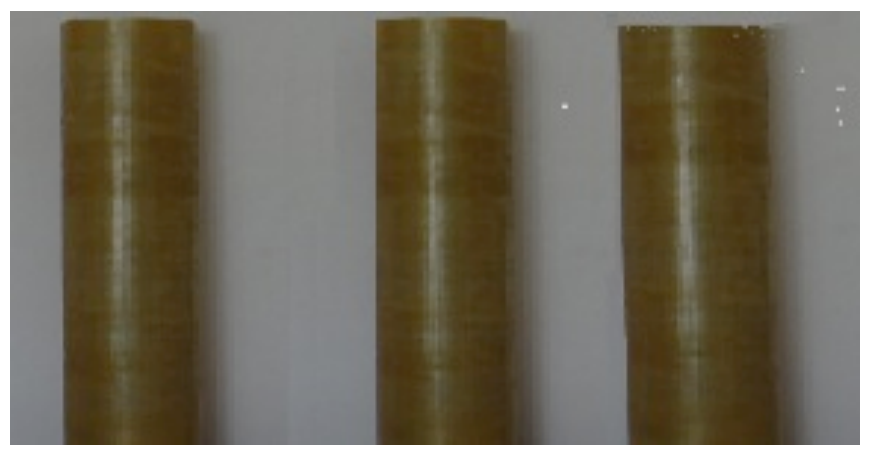

Fig. (2). Multi-directional filament winding material tubes.

\subsection{Radial Pressure Experiment}

In order to apply static compression tests, specimens used in the experiment are cylindrical specimens intercepted from the tubes with the length of $200 \mathrm{~mm}$, and also both ends are intensively protected to prevent experimental bias caused by edge damage.

This paper uses the AG250KN Shimazu, a Japanese omnipotent material testing machine, to radially compress the multi-directional filament winding composite tube, as shown in Fig. (3), under room temperature conditions, the loading rate is $5 \mathrm{~mm} / \mathrm{min}$; using VS9002RIC (Vallen highaccuracy acoustic emission sensor) sensor, the frequency range is between 100 and $900 \mathrm{kHz}$. The system collects waveform data to 4,096 points, and sets 8-channel to gather acoustic emission signal (Schematic illustration of $\mathrm{AE}$ detecting system is given in Fig. 4) and the output signals of the sensor are amplified through it and then transferred to the host machine through a long cable for processing. Its main role is: high-impeditive sensor and low-impeditive transmission cables match to minimize signal attenuation; suppressing cable noise by amplifying weak

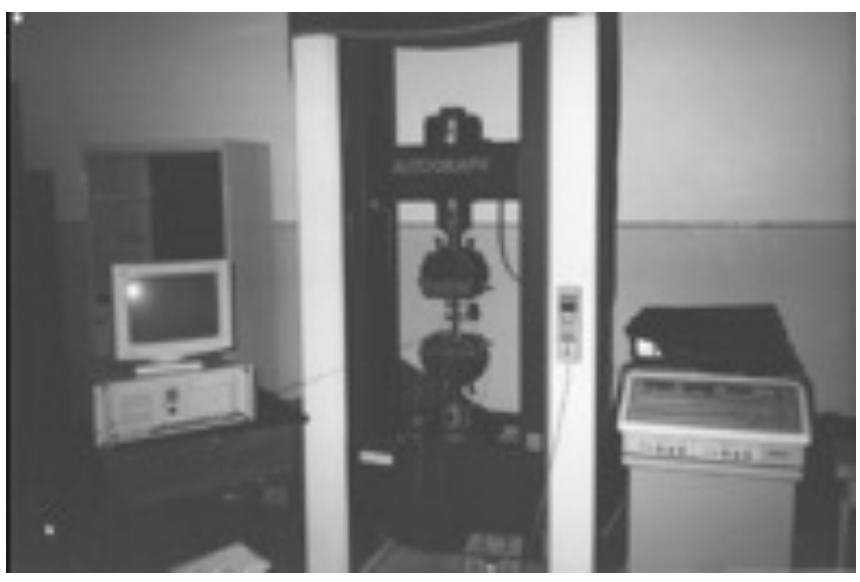

Fig. (3). AG250KN universal material experiment machine.

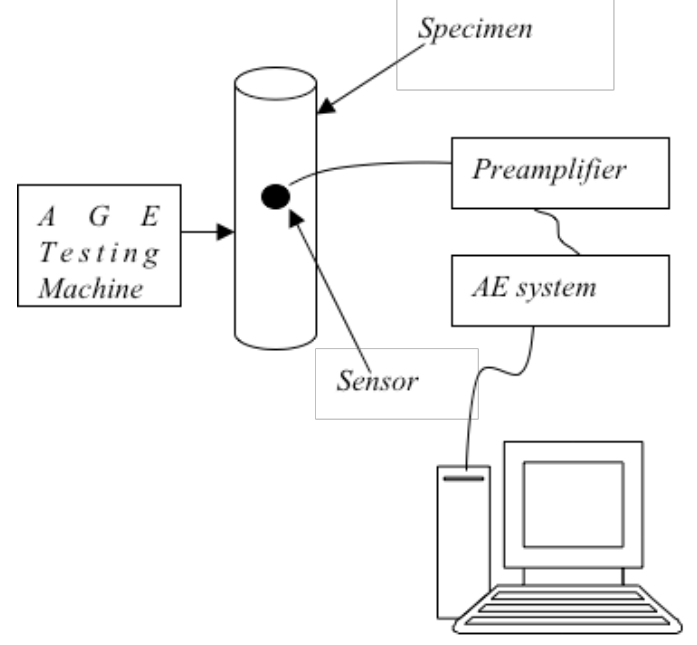

Data processing

Fig. (4). Schematic illustration of AE detecting system.

Table 1. Laminated structure and geometrical parameters of specimen.

\begin{tabular}{|c|c|c|c|c|}
\hline Laminated Structure & Yarn Width $(\mathbf{m m})$ & Internal Diameter $(\mathbf{m m})$ & Fiber Volume Fraction / \% & Plies \\
\hline \hline$\left(90^{2}, 0, \pm 70.07\right)^{2}$ & 9 & $\Phi 100$ & 39.55 & 10 \\
\hline
\end{tabular}

PS: the subscript ' 2 ' in the laminate means 2 circles. 
input signals in order to improve the signal noise ratio; and providing frequency filter. Main amplifier and filter are important components of the system, while the former provides further amplification for acoustic emission signal so as to carry on signal processing for subsequent parameter measurement and calculation unit. It is equipped with adjustable magnification, enabling the gain of the whole system to reach to $60 \sim 100 \mathrm{~dB}$. Filters added in the detection system are mainly used to exclude noise and restrict the frequency range of the inspection system, so that the system could be accommodated to detect in complex and noisy environment. After being processed by the amplifier and filter, signals are converted into digital form via APD converter, and then will be further analyzed by computer. The AE signal processing chain is shown in Fig. (5).

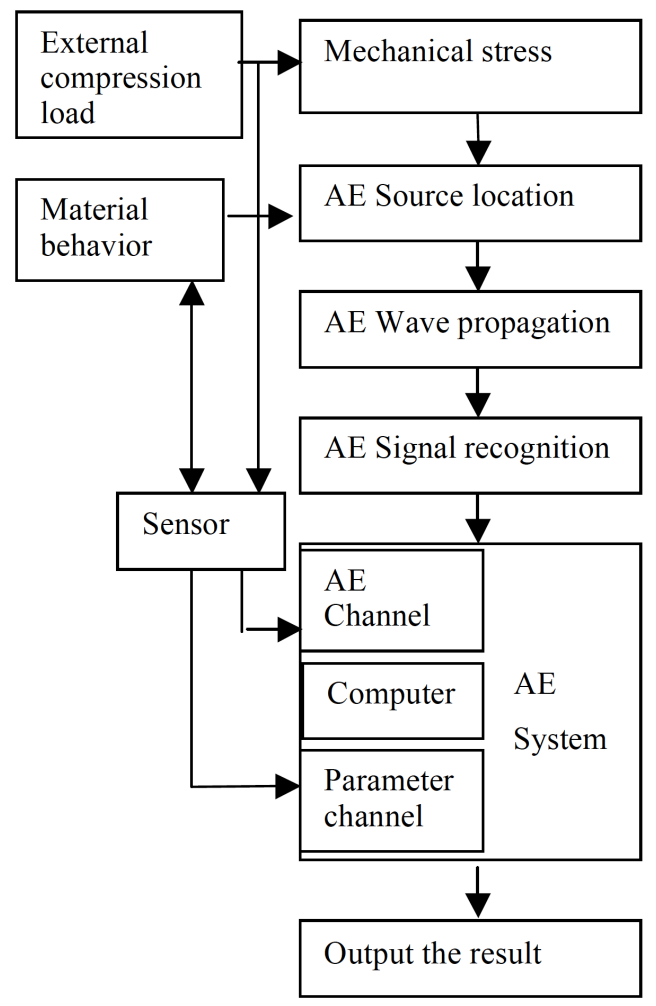

Fig. (5). AE process chain.

\subsection{Acoustic Emission Wavelet Transform}

The precise definition for wavelet function is: $\phi(t) \in L^{2}(R)$ is the square integrable function, if its Fourier transform $\hat{\phi}(\omega)$ satisfies the condition:

$c_{\phi}=\int_{R} \frac{\left|\hat{\phi}(\omega)^{2}\right|}{|\omega|} d \omega<\infty$

$\phi(t)$ can be referred to as a basic wavelet or mother wavelet. Equation (1) is the permissible condition for wavelet function. After stretching and translating, the mother wavelet $\phi(t)$ generates:

$\phi_{a b}(t)=\frac{1}{\sqrt{|a|}} \phi\left(\frac{t-b}{a}\right) a, b \in R ; a \neq 0$
In the Equation (1): $\mathrm{a}$ is a scaling factor; $\mathrm{b}$ is the translating factor, known as the $\phi_{a b}(t)$ wavelet function that depends on the parameters a, b. Because a and b are continuously changing parameters, the term $\phi_{a b}(t)$ is called continuous wavelet. Expanding functions $f(t)$ in the $L^{2}(R)$ space under the wavelets, it can be called the continuous wavelet transform of a function $f(t)$ its expression is:

$W_{f}(a, b)=\frac{1}{\sqrt{|a|}} \int_{R} f(t) \phi^{*}\left(\frac{t-b}{a}\right) d t$

The reconstruction formula (inverse transform) is:

$f(t)=\frac{1}{C_{\phi}} \int_{-\infty}^{\infty} \int_{-\infty}^{\infty} \frac{1}{a^{2}} W_{f}(a, b) \phi\left(\frac{t-b}{a}\right) d a d b$

In practical use, especially on computer, continuous wavelet must be discretized. Typically, the discretizing formula for continuous scaling factor a and translating factor is: $a=a_{0}^{j}, b=k a_{0}^{j} b_{0} j \in Z$, where $a_{0}, b_{0}$ is the extension step, and the corresponding discrete wavelet based $a_{j, k}(t)$ is:

$\phi_{j, k}(t)=a_{0}^{\frac{1}{j}} \phi\left(a_{0}^{-j} t-k b_{0}\right)$

The expression of the function $f(t)$ discrete wavelet transform is:

$W_{f}(j, k)=\int_{R} f(t) a_{0}^{-\frac{j}{2}} \phi^{*}\left(a_{0}^{-j} t-k b_{0}\right) d t$

\section{TEST RESULTS AND ANALYSIS}

\subsection{Analysis of AE Signals}

Fig. (6) shows the curve of the cumulative number of $\mathrm{AE}$ hits and loading time. In the initial loading stage, the number of $\mathrm{AE}$ hits is grows rarely and the cumulative number of $\mathrm{AE}$ hits grows slowly. The number of AE hits during the late period of the fatigue damage test increases rapidly. The surface of the specimen is damaged more and more seriously, and the density of the number of AE hits increases rapidly. The component is thus, quickly destroyed.

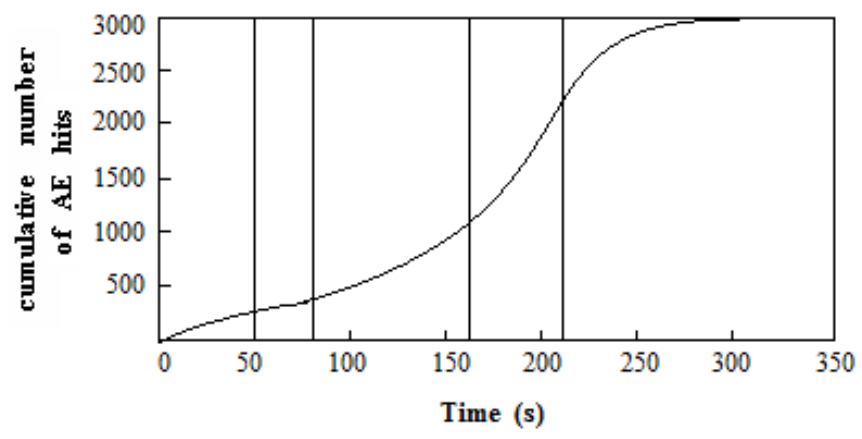

Fig. (6). Cumulative number of AE hits.

The acoustic emission signal amplitudes of multidirectional filament winding specimens during radial compression testing change along with time variation curve are shown in Fig. (7). Acoustic emission signals collected in the test are decomposed and reconstructed with $\mathrm{db} 5$ wavelet, 
and Figs. (8-12) are the frequency distribution charts after the signals are decomposed by damage patterned wavelet packet. According to frequency distribution charts of acoustic emission signal after wavelet transformation, the duration of its high frequency energies peak is much shorter than its low frequency energy peak, while the duration for releasing energy during damage is actually the reflection of the ductility or brittleness of the material. High frequency energy corresponds to brittle damage, and low frequency energy is more likely to be related to ductile damage. Cellosilk breakage is the most brittle damage in fiber composites, and the cracking of matrix resin tends to endure relatively longer than cellosilk breakage. Therefore, signals during high frequency and short duration should correspond to the cellosilk breakage; low frequency and long duration correspond to the destruction of the matrix resin, while middle frequency may be connected with the delamination damage of cellosilk and matrix material on the ground of the time-frequency charts.

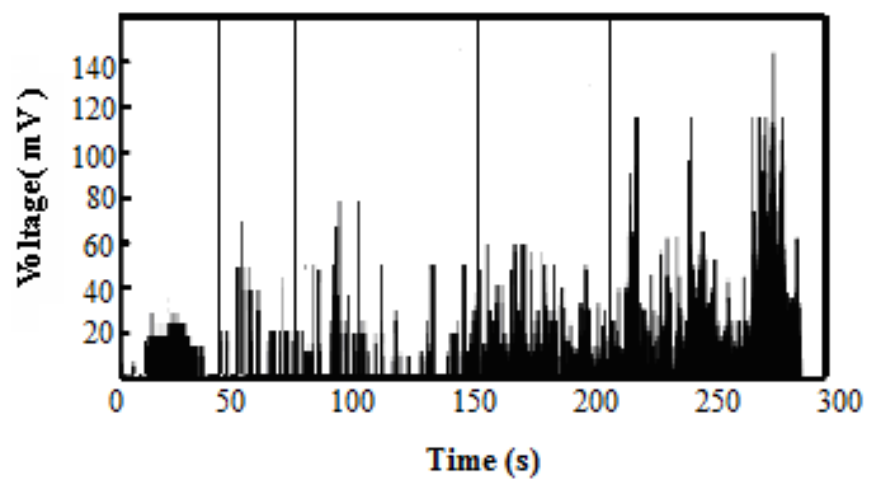

Fig. (7). AE signal amplitudes.

\subsection{Analyzing the Evolution Process of the Specimen Damage}

Analyzing the evolution process of multi-directional fiber winding tube damage by stages:

\subsubsection{Phase I}

During the first phase $(0-50 \mathrm{~S})$, phase displacement is small, and cumulative number of impacts does not change much. Fig. (7) indicates that the acoustic emission amplitudes are generally smaller than $60 \mathrm{~dB}$ at this stage, while the released energy is very low and the duration is less than 1s. It is mainly the influence of noise in experimental system and the result of mild matrix crack. There also appears some signal amplitudes that are greater than $60 \mathrm{~dB}$ in this phase, and it is possibly due to the fact that winding fiber itself has ease allowance when the tension is uneven in the winding process, and fiber has the tendency to shrink after deforming and cooling down under pressure at high temperature. So internal stress is formed between fiber inside the material and matrix, and it produces a great amount of deformation energy after damage, characterized by large amplitude of the acoustic emission signal. During the first loading phase, the maximum displacement turns from 0 to $4.5 \mathrm{~mm}$, receiving 242 acoustic emission signals. Most energy peaks of the acoustic emission signals are centralized within the middle frequency $(160-300 \mathrm{kHz})$ and the low- frequency $(80-160 \mathrm{kHz})$ range. As shown in Fig. (7), high frequency energy peaks are very few.

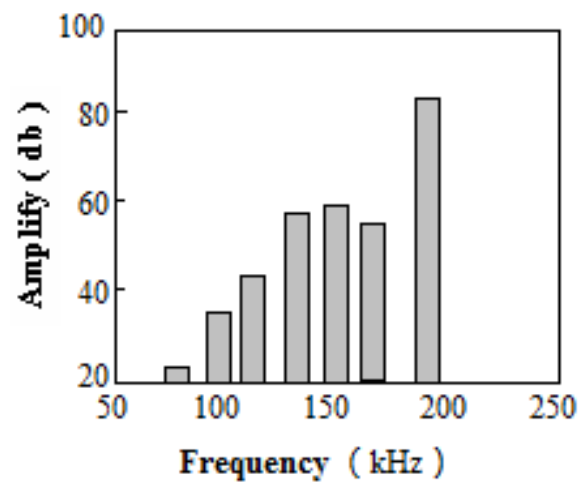

Fig. (8). AE signal bandwidth frequency distribution during the first phase.

\subsubsection{Phase II}

During the second phase (51-85 seconds), the cumulative number of impacts grows very fast, but the total number is very small while the damage is on the rise. The acoustic emission signals with the amplitude of $60 \sim 80 \mathrm{~dB}$ are on the rise, the duration is around $1-2 \mathrm{~s}$, and energy has also boosted significantly. At this stage, compressed center shifts reach up to $6.7 \mathrm{~mm}$, receiving a total of 53 acoustic emission signals, and the corresponding frequency range is between 100 and $300 \mathrm{kHz}$, medium-frequency and low-frequency energy peaks of acoustic emission signals continue to dominate as shown in Fig. (8). However, the damage progresses slowly as the number of acoustic emission signals are few. Damage at this stage mainly comes from the further propagation of cracks formed in the first phase. Therefore, the fiber and matrix are separated at the front of the cracking edge, causing interfacial debonding.

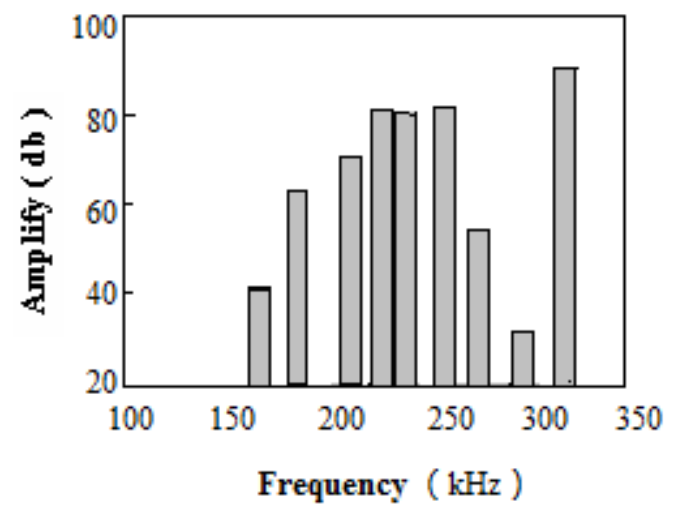

Fig. (9). AE signal bandwidth frequency distribution during the second phase.

\subsubsection{Phase III}

The third phase (86-160 seconds) starts when the cumulative number of impacts grows from slow to fast with a large sum. The amplitudes at this stage can be greater than $80 \mathrm{~dB}$, and even above $90 \mathrm{~dB}$. Energy and duration are also on the rise, and the duration is greater than $3 \mathrm{~s}$. As we can see from Fig. (9), middle and low frequency energy peaks still remain to exist, and high frequency energy peaks (over $300 \mathrm{kHz}$ ) of acoustic emission signals also begin to increase 
rapidly. This indicates that matrix cracking exacerbates and partial crack propagates unstably. Owing to the small plasticity of matrix materials, they completely crack before the fiber breaks down, resulting in the obvious decrease of acoustic emission impacts in the later process of this stage. With further increase of loading, fiber is pulled out from the matrix, and parts of it even begin to fracture. It then forms great stress concentration around the fracture and transfers the stress released by broken fiber to its adjacent fibers, causing the successive fracture of adjacent fibers. This damage occurs mainly on the interface between fiber and matrix and fracture surface of the matrix, leading to the significant relieving of partial stress concentration and concave slope on the surface of specimen.

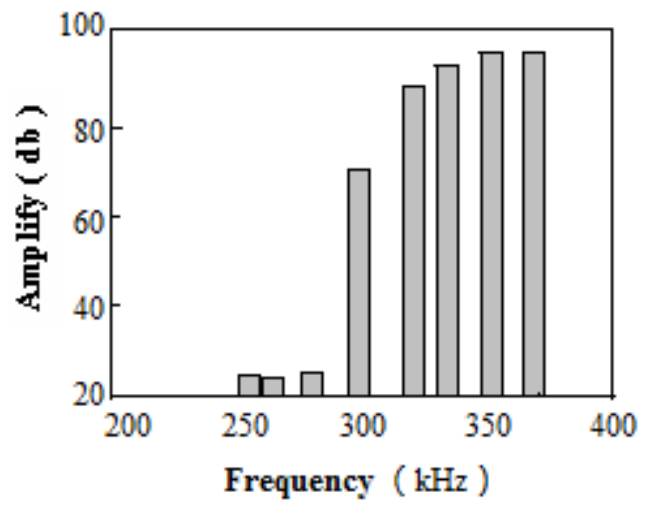

Fig. (10). AE signal bandwidth frequency distribution during the third phase.

\subsubsection{Phase IV}

In the IV stage (around 161-210 seconds), the amplitude value range of acoustic emission signals increases greatly compared with the last stage, the average value can reach over $90 \mathrm{~dB}$, and the number of acoustic emission signals significantly soars, receiving 664 acoustic emission signals altogether. In light of Fig. (10), high frequency components of acoustic emission signals continues to grow, and energy peaks of higher frequency (greater than $400 \mathrm{kHz}$ ) will take place, energy peaks of acoustic emission signals also have obvious growth. It signifies that materials have various damage patterns, and the joint surface fractures between fascicules, and between fascicules and matrix, due to which the crack begins to expand.

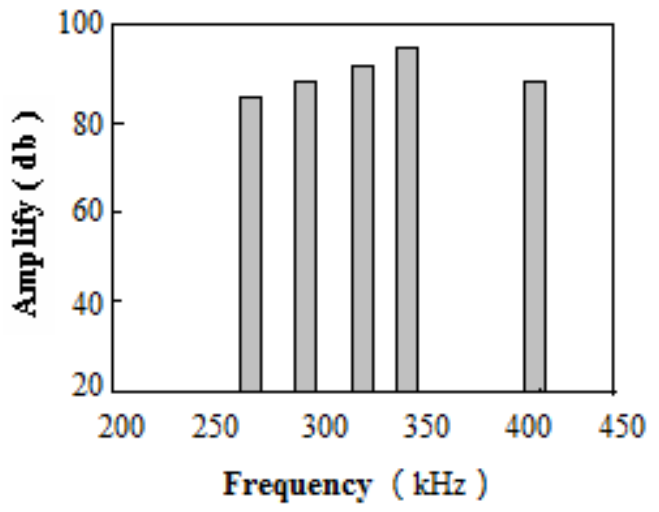

Fig. (11). AE signal bandwidth frequency distribution during the fourth phase.

\subsubsection{Phase $V$}

The fifth stage (210 seconds above) is the last stage of loading fatigue on the specimen, the acoustic emission range on this stage can reach to $100 \mathrm{~dB}$, acoustic emission energy could achieve the maximum value, and the duration is over $10 \mathrm{~s}$, receiving 981 acoustic emission signals in total. According to Fig. (11), the energy peaks of acoustic emission signals become very intensive, and band width covers almost every possible frequency range, which indicates that the specimen receives different types of damages in the last loading stage and its bearing capacity sharply declines, showing that there are lots of fiber fractures and interfacial separation, the specimen becomes completely ineffective (Fig. 12).

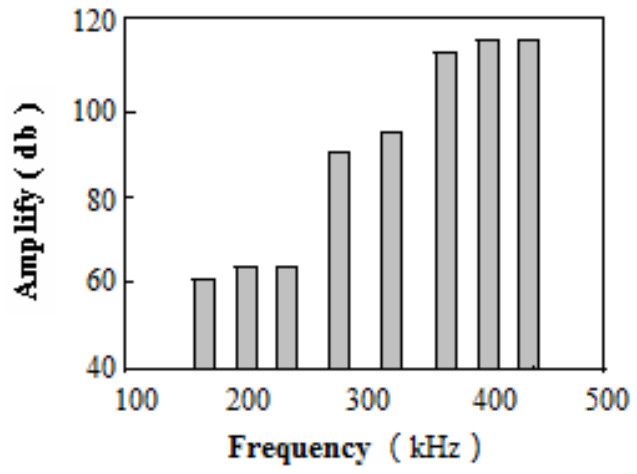

Fig. (12). AE signal bandwidth frequency distribution during the fifth phase.

\section{CONCLUSION}

As continuously exerting radial external pressure on the multi-directional filament winding composite tube, the amplitude value of the acoustic emission signals released by the specimen is on an increasing trend, the number of cumulative events of acoustic emissions also continues to increase, and the density for the number of acoustic emission events per unit time grows continuously. Some noises are filtered out through wavelet transforming acoustic emission signals. According to the frequency distribution of acoustic emission signal diagram, one acoustic emission signal often includes damage signals with different frequencies, representing different damage patterns. The damage pattern represented by high frequency signals is the extremely brittle failure, while the damage model of low frequency signals is relatively the ductile failure.

\section{CONFLICT OF INTEREST}

The authors confirm that this article content has no conflict of interest.

\section{ACKNOWLEDGEMENTS}

Declared none.

\section{REFERENCES}

[1] Lindley TC, Palmer IG, Richards CE. Acoustic emission monitoring of fatigue crack growth. Mater Sci Eng 1978; 32(1):115 . 
[2] Carpinteri A, Lacidogna G, Pugon N. Structural damage diagnosis and life-time assessment by acoustic emission monitoring. Eng Fract Mach 2007; 74(1/2): 273-89.

[3] Tandon N, Choudhury A. A review of vibration and acoustic measurement methods for the detection of damages in rolling element bearings. Tribol Int 1999; 32(8): 469-80.

[4] Tan CK, Mba D, Identification of the acoustic emission source during a comparative study on diagnosis of a spur gearbox. Tribol Int 2005; 38(5): 469-80.

[5] Mechan RL, Mullin JV, Analysis of composite failure mechanisms using acoustic emissions. Compos Mater 1971; 5: 266-9.

[6] Johnson M, Gudmundson P. Broad band transient recording and characterization of acoustic emission events in composite laminates. Composites Sci Technol 2000; 60(15): 2803-18.

[7] Amilcar Q, Basir S, Frederick J. Acoustic emission based tensile characteristics of sandwich composites. Eng 2004; 35(6): 563-71.
[8] Dzenis YA, Qian J. Analysis of microdamage evolution histories in composites. Int J Solodsamd Struct 2001; 38(10): 1821-54.

[9] Chou HY, Mouritz AP. Acoustic emission analysis of composite pressure vessels under constant and cyclic pressure. Composites 2015; 70(15): 111-20.

[10] Giovanni P, Frode G, Steinar S. Numerical/experimental impact events on filament wound composite pressure vessel. Composites 2015; 69(14): 406-17.

[11] Mesut Uyaner, Memduh Kara, Aykut Sahin. Fatigue behavior of filament wound E-glass/epoxy composite tubes damaged by low velocity impact. Composites Part B 2014; 61: 358-64

[12] Martins LA, Bastian TA. Netto reviewing some design issues for filament wound composite tubes. Mater Design 2014; 24(9): 242-9.

(C) Hua and Cheng; Licensee Bentham Open.

This is an open access article licensed under the terms of the Creative Commons Attribution Non-Commercial License (http://creativecommons.org/licenses/ by-nc/4.0/) which permits unrestricted, non-commercial use, distribution and reproduction in any medium, provided the work is properly cited. 